PAPER

\title{
A syndromal analysis of neuropsychological outcome following coronary artery bypass graft surgery
}

\author{
A C Kneebone, M A Luszcz, R A Baker, J L Knight
}

J Neurol Neurosurg Psychiatry 2005;76:1121-1127. doi: 10.1136/jnnp.2004.047456

See end of article for authors' affiliations

author' affiliations

Correspondence to: Dr Anthony C Kneebone, Department of

Psychological Medicine, Flinders Medical Centre, Bedford Park 5042,

Australia; tony.kneebone@

fmc.sa.gov.au

Received 10 June 2004

Revised version received

24 November 2004

Accepted

24 November 2004
Background: Studies of neuropsychological outcome following coronary artery bypass graft surgery $(C A B G)$ have traditionally dichotomised patients as "impaired" or "unimpaired". This conceals the potential heterogeneity of deficits due to different mechanisms and sites of brain injury.

Objectives: To explore neuropsychological outcome following CABG and determine to what extent it conforms to prototypic cortical and/or subcortical neurobehavioral syndromes and whether different intraoperative physiologic measures are associated with different subtypes of neuropsychological outcome.

Methods: Neuropsychological tests were administered to 85 patients before and after elective CABG and to 50 matched normal control subjects. Pre- to postoperative change scores were computed using standardised regression based norms. Change scores on selected memory measures were subjected to cluster analysis to identify qualitatively distinct subtypes of memory outcome. Emergent clusters were compared on non-memory measures, intraoperative physiologic measures, and demographic variables. Results: Three subtypes of memory outcome were identified: memory spared ( $48 \%$ of patients), retrieval deficit (35\%), and encoding/storage deficit (17\%). Contrary to expectation, the subgroups were indistinguishable on measures of confrontation naming and manual dexterity and on intraoperative cardiac surgical physiologic measures and demographic variables. The encoding/storage deficit subgroup exhibited executive dysfunction.

Conclusions: Heterogeneous profiles of neuropsychological dysfunction were found following CABG although they did not tightly conform to prototypic cortical and subcortical neurobehavioral syndromes. This challenges the value and appropriateness of the common practice of collapsing individual test scores to arrive at a single figure to define "impairment". Whether different subtypes of neuropsychological outcome are caused by different pathophysiologic mechanisms remains unknown.
$\mathrm{N}$ systematic research has attempted to define the uality of neuropsychological changes following coronary artery bypass graft surgery (CABG). Instead, neuropsychological research has largely focused on defining the incidence of postoperative impairment by dichotomising patients as "impaired" or "unimpaired". ${ }^{1}$ This dichotomy does not permit investigation of potential subtypes of adverse neuropsychological outcome which may be the product of different mechanisms of injury impacting upon different brain structures.

Histopathologic and MRI studies have demonstrated the vulnerability of specific brain regions to intraoperative injury in cardiac surgery employing cardiopulmonary bypass (CPB). Early postmortem studies emphasised the role of anoxia in causing injury to arterial borderzone regions, in particular the hippocampi. ${ }^{2-4}$ More recently, microemboli have been considered the likely agent of brain injury and postoperative neuropsychological dysfunction with the most common sites of MRI defined infarction being within the deep subcortical white matter, basal ganglia, caudate nucleus, and corona radiata. ${ }^{5-11}$ Given these findings, there is a rational basis to anticipate that $\mathrm{CABG}$ might impart qualitatively different neuropsychological outcomes across individuals which are dependent upon the primary sites and mechanisms of injury.

A theoretic framework for conceptualising potential patterns of neuropsychological outcome following CABG is that of "cortical" versus "subcortical" neurobehavioral syndromes. ${ }^{12} 13$ The link between these syndromes and cardiac surgery relates to hypothetical commonalities in the primary sites of neuropathology. Specifically, in dementia of the Alzheimer type (DAT), a prototypic cortical neurobehavioral syndrome, there is early prominent involvement of temporal lobe structures including the hippocampi. ${ }^{14}$ On the other hand, in subcortical syndromes such as vascular dementia (VaD) there is prominent involvement of the deep white matter, basal ganglia, and thalamus, ${ }^{12} 1516$ which are the same structures considered susceptible to microembolic injury in CABG.

Cortical and subcortical syndromes are dissociable in terms of spared and impaired cognitive functions found in each (table 1). Specifically, on tests of episodic memory, such as the California Verbal Learning Test (CVLT), patients with DAT demonstrate deficient free recall and recognition memory. ${ }^{13}{ }^{16-19}$ This has been interpreted as a breakdown in memory encoding and storage. ${ }^{13}$ In contrast, patients with subcortical disorders demonstrate impaired free recall with relative preservation of recognition memory suggesting a breakdown in search and retrieval mechanisms. ${ }^{13}{ }^{16-19}$ On non-memory tasks, confrontation naming is frequently inferior in DAT patients compared to subcortical patients due to a breakdown in semantic processing thought to be mediated by the temporal neocortex. ${ }^{20}$ On the other hand, subcortical groups are generally inferior on tests of executive function, possibly due to functional disconnection of the

Abbreviations: BNT, Boston Naming Test; CABG, coronary artery bypass graft surgery; COWAT, Controlled Oral Word Association Test; CPB, cardiopulmonary bypass; CVLT, California Verbal Learning Test; DASS, Depression Anxiety and Stress Scales; DAT, dementia of the Alzheimer type; DSym, Wechsler Adult Intelligence Scale-Revised Digit Symbol subtest; NART-R, National Adult Reading Test-Revised; PegsRL, Purdue Pegboard simultaneous right and left hand trial; SRB, standardised regression based; TMT, Trail Making Test; VaD, vascular dementia 
frontal lobes from underlying subcortical structures. ${ }^{21}$ Finally, disorders of movement and reduced psychomotor speed are hallmarks of subcortical disorders due to variously placed lesions within the basal ganglia-thalamocortical motor circuit, ${ }^{12}{ }^{22}$ with VaD patients being distinguished from DAT patients by their inferior performances on measures of manual dexterity and motor performance..$^{23}$

Using cluster analysis, this study explores whether different profiles of neuropsychological outcome occur following $\mathrm{CABG}$ and to what extent they conform to prototypic cortical and/or subcortical neurobehavioral syndromes. Assuming that the emergent clusters conform to one or both these syndromes, it is hypothesised that they will be distinguishable on the basis of intraoperative physiologic measures. Specifically, the cortical subgroup will have been subjected to comparatively lower intraoperative cerebral perfusion pressure and flow rates resulting in hypoxic brain injury maximally involving the hippocampi. Alternately, patients within the subcortical subgroup will have been subjected to comparatively greater intraoperative microembolic insult as a result of a higher number of perfusionist interventions ${ }^{25}$ and possibly longer CPB time. ${ }^{1126}$

\section{METHODS}

\section{Patients}

From 142 consecutive patients who underwent elective CABG performed by a single surgeon (JLK) between January 23, 1996 and December 12, 1997, a final sample of 85 patients was obtained. Study inclusion criteria included English as a first language and no past history of cardiac or carotid artery surgery, neurologic disease/trauma, psychiatric illness requiring hospitalisation, or substance abuse. Of the 57 excluded patients, 29 were lost to 6 month follow up due to long distance travel difficulties, 20 declined participation, four were either deceased or suffered postoperative stroke, and four had illnesses unrelated to CABG preventing follow up. All patients provided informed consent to participate in this institutionally approved study (Flinders Medical Centre Clinical Investigation Committee approval number 136/94).

\section{Control subjects}

Fifty unpaid volunteers were recruited from senior citizens groups and the Flinders Medical Centre Volunteers Service. Each met the same study inclusion criteria as the CABG patients. The demographic and baseline neuropsychological data for both patients and controls are presented in table 2 . Except for patients having a significantly higher anxiety level at baseline assessment, there were no between group differences on any variable. Importantly, amongst the patients baseline neuropsychological performance was found to be independent of mood state, the highest correlation being between depression and CVLT \%SDFR (SDFR, short delay free recall; \%SDFR, $($ SDFR/trial 5) $\times 100)(\mathrm{r}=-0.18$, $\mathrm{p}=0.06$ ).

Table 1 Neuropsychological features of cortical and subcortical syndromes

\begin{tabular}{lll}
\hline \multirow{2}{*}{$\begin{array}{l}\text { Neuropsychological } \\
\text { domains }\end{array}$} & \multicolumn{2}{l}{ Behavioural syndrome } \\
\cline { 2 - 3 } & Cortical & Subcortical \\
\hline $\begin{array}{lll}\text { Episodic memory } \\
\text { Delayed free recall }\end{array}$ & Impaired & Less impaired \\
$\begin{array}{l}\text { Recognition memory } \\
\text { Confrontation naming }\end{array}$ & Impaired & Spared \\
Executive function & Spared/less impaired & Impaired \\
Psychomotor function & Spared/less impaired & Impaired \\
\hline
\end{tabular}

\section{Surgical technique}

Patients were randomly assigned to undergo CABG using either moderately hypothermic or normothermic CPB. Bypass was instituted following cannulation of the aorta and the right atrium utilising non-pulsatile perfusion and alpha stat $\mathrm{pH}$ management. A standard PVC tubing circuit (Cobe Laboratories, Anaheim, CA), $40 \mu \mathrm{m}$ arterial line filter (Medtronic, Anaheim, CA), and Maxima membrane oxygenator (Medtronic) were used in all cases. All patients received blood cardioplegia delivered anterograde, to induce asystole. The hypothermic group were cooled to 30 or $32^{\circ} \mathrm{C}$ (all systemic temperatures were measured at the nasopharynx) and received cold blood cardioplegia $\left(8^{\circ} \mathrm{C}\right)$. The target rewarming rate for the hypothermic patients was a nasopharyngeal temperature increase of $\leqslant 0.5^{\circ} \mathrm{C} / \mathrm{min}$, with a $10^{\circ} \mathrm{C}$ maximal gradient between arterial and venous blood temperature. The actual average rewarming rate was $0.3^{\circ} \mathrm{C} /$ min, and the maximum rewarming rate was $0.7^{\circ} \mathrm{C} / \mathrm{min}$. The normothermic group were maintained at normal body temperature $\left(36-37^{\circ} \mathrm{C}\right)$ and received warm blood cardioplegia $\left(36-37^{\circ} \mathrm{C}\right)$. In addition cardioplegia was delivered at the conclusion of each distal graft. All subjects were managed within institutional perfusion guidelines including maintaining haemoglobin levels above 7 during the bypass period, $\mathrm{PaO}_{2}$ 150-250 mm Hg, $\mathrm{Paco}_{2} 35-45 \mathrm{~mm} \mathrm{Hg}$, pH 7.35-7.45.

\section{Neuropsychological examination}

Qualified examiners tested patients the day before operation, the day before discharge, and 6 months post-discharge. Control subjects were tested three times across the same intervals. Only the baseline and 6 month data are reported in this study because the Statement of Consensus on Assessment of Neurobehavioral Outcomes After Cardiac Surgery $^{27}$ considers data obtained within the immediate postoperative period to be unstable and influenced by extraneous factors including sleep deprivation, anaesthetic effects, ${ }^{28}$ pain, ${ }^{29}$ and other general effects of surgery. ${ }^{8}$ Additionally, it was felt that any longer term effects of CABG surgery were likely to be more meaningful to patients' everyday functioning.

Neuropsychological test selection was substantially based on the Statement of Consensus. ${ }^{27}$ The test battery in order of administration was CVLT, ${ }^{30}$ Purdue Pegboard (simultaneous right and left hand trial; PegsRL), ${ }^{31}$ Controlled Oral Word Association Test (COWAT), ${ }^{32}$ Trail Making Test (TMT), ,33 Boston Naming Test (BNT), ${ }^{34}$ Wechsler Adult Intelligence Scale-Revised Digit Symbol subtest (DSym), ${ }^{35}$ National Adult Reading Test-Revised (NART-R; administered only at baseline), ${ }^{36}$ and the Depression Anxiety and Stress Scales (DASS). ${ }^{37}$

Cluster analysis was performed using the two measures of the CVLT that most readily distinguish cortical and subcortical subtypes of neuropsychological dysfunction, these being short delay free recall after interference (\%SDFR) and recognition memory (Recognition). ${ }^{13} \%$ SDFR was calculated by dividing the number of words correctly free recalled by the total number of words correctly recalled on the last learning trial $(\times 100)$. Every second patient or control subject was administered the alternate form of the CVLT at baseline with the same form being administered at 6 month follow up. All tests were administered and scored in the prescribed manner with the exception of the TMT in which the ratio of the time taken to complete part $\mathrm{B}$ relative to the time taken to complete part A was calculated (that is, part B seconds/part A seconds). This ratio measure has been shown to provide a purer measure of mental flexibility independent of overall speed. $^{38}$ 
Table 2 Demographic, surgical, and baseline neuropsychological data for patients and control subjects

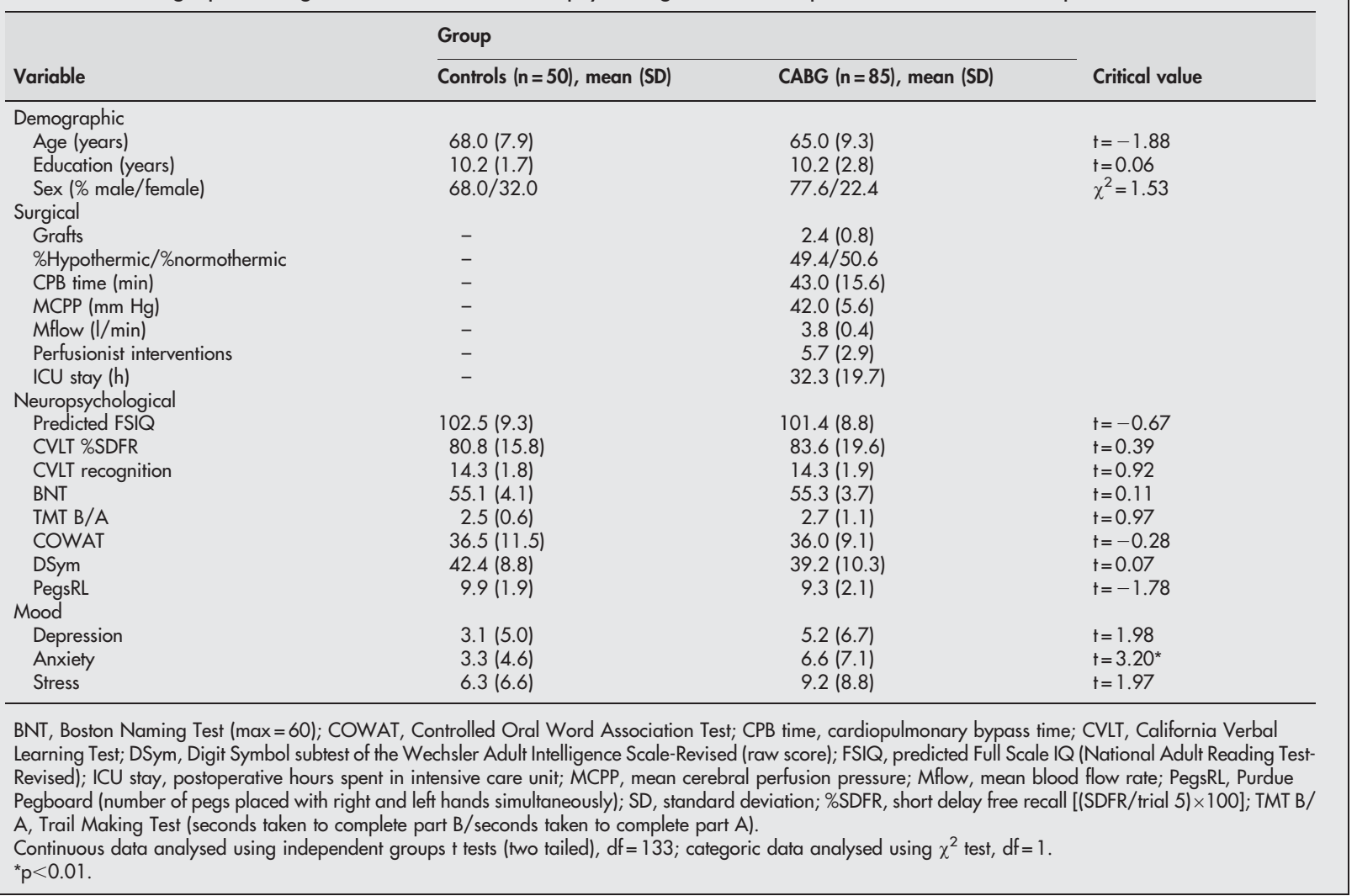

\section{Definition of neuropsychological change}

Standardised regression based (SRB) methodology was employed to quantify the magnitude of pre- to postoperative change on neuropsychological measures independent of measurement error, practice effects, and regression to the mean. ${ }^{39}$ This methodology entailed 6 month retest scores of control participants being regressed against baseline scores and other potential predictors of retest performance (that is, age, sex, years' education, predicted NART-R Full Scale IQ, and test-retest interval). These regression equations were then used to obtain predicted retest scores for individual CABG surgery patients on each neuropsychological measure. The differences between the observed and predicted retest scores were transformed into standardised $\mathrm{Z}$ scores (SRB change scores) as follows: $\mathrm{Z}$ score $=\left(\mathrm{Y}_{\mathrm{O}}-\mathrm{Y}_{\mathrm{p}}\right) / \mathrm{SE}_{\mathrm{reg}}$, where $\mathrm{Y}_{\mathrm{O}}$ is the observed retest score, $\mathrm{Y}_{\mathrm{p}}$ is the predicted retest score, and $\mathrm{SE}_{\mathrm{reg}}$ is the standard error of the estimate from the regression equation (that is, SD of the residuals). Consistent with the normal distribution of $\mathrm{z}$ scores, any SRB change score exceeding \pm 1.64 (90\% confidence interval) is statistically rare and indicates significant improvement or decline thus enabling categoric classifications of outcome for each test measure if desired. However, in this study for the data to be subjected to cluster analysis the derived SRB z scores for each test measure were retained as continuous data reflecting the magnitude of pre- to postoperative change.

\section{Cluster analysis}

Given the consistently proven validity of differential performance on CVLT measures \%SDFR and Recognition in distinguishing cortical and subcortical syndromes, ${ }^{13}{ }^{17-19}$ the SRB $\mathrm{z}$ scores for these measures were subjected to cluster analysis. Tests tapping executive and psychomotor functions were subsequently used for external validation of the cluster solutions.
Three hierarchical agglomerative methods of clustering were employed (average linkage, complete linkage, and Ward's method) using squared Euclidian distance as the similarity/dissimilarity measure. ${ }^{40}$ The number of clusters derived from each clustering method was objectively established using Mojena's method. ${ }^{41}$ To validate the cluster solutions, the data were subjected to k-means iterative partitioning. Checks for internal validity using misclassification analysis and split-sample replication were carried out. ${ }^{40}$ The cluster method with the highest level of internal validity was selected as the final solution.

Between group differences on demographic, neuropsychological, and cardiac surgical data measures were tested using one way ANOVA (post hoc Tukey's HSD) or Kruskal-Wallis tests (post hoc Mann-Whitney U tests) depending on the equality of cell sizes and/or heteroskedasticity and distribution shape. $\chi^{2}$ tests were used for categoric data. Pearson and Spearman correlations were used to examine the relationships between neuropsychological change scores and medical and cardiac surgical variables

\section{RESULTS}

\section{Regression analyses}

Regression equations derived from control data used to predict individual patient's 6 month postoperative score on each neuropsychological measure are presented in table 3. Baseline performance entered as a predictor of retest performance on all neuropsychological measures, accounting for between 13\% (TMT B/A) and 74\% (DSym) of the variance $(\mathrm{p}<0.05)$. Age entered as a significant predictor of DSym only, accounting for $1.7 \%$ of the variance with younger age predicting a higher retest score. Gender entered as a predictor of retest performance on PegsRL accounting for $5.9 \%$ of the variance with female gender being associated with a higher level of retest performance. Years of education, FSIQ, and 
test-retest interval did not enter as predictors of retest performance on any of the neuropsychological measures.

The regression equations generated from the control sample were applied to the data of individual CABG patients in order to obtain their predicted retest scores on each neuropsychological measure. The differences between the observed and predicted retest scores were calculated and transformed into standardised SRB z scores for subsequent analyses.

\section{Cluster analyses}

Subjecting SRB z scores on \%SDFR and Recognition to the hierarchical agglomerative cluster methods produced either a two or three cluster solution. Thus, subsequent k-means iterative partitioning was performed with both two and three group solutions being requested. Internal validation revealed the three group k-means solution to be the most stable with a mean concordance across clustering methods of $88.2 \%$ and split-sample concordance of $97.7 \%$ and $100 \%$ with the parent sample. The three group k-means solution was therefore used in subsequent analyses.

\section{Analyses of cluster subtypes}

Table 4 shows cluster l comprised 41 patients (48.2\%), cluster 2 comprised 30 patients $(35.3 \%)$, and cluster 3 comprised 14 patients (16.5\%). To reduce the subjectivity interpreting the profiles of SRB $\mathrm{z}$ scores on attribute variables, the scores obtained by each cluster were compared to those of the control subjects.

As shown in table 4, the mean SRB z scores on \%SDFR and Recognition obtained by cluster 1 did not significantly differ from the control group. Thus, this cluster comprised patients who did not demonstrate a significant postoperative decline (or improvement) in memory function and were labelled the "Memory spared" cluster. Cluster 2 demonstrated impaired delayed recall (\%SDFR) and preserved recognition memory relative to the control group. This profile is consistent with a memory retrieval deficit typically associated with subcortical pathology. Finally, cluster 3 demonstrated impaired delayed recall and recognition memory relative to the control group consistent with a memory encoding/storage deficit in which hippocampal pathology is considered to predominate. Effect sizes (Cohen's d) representing the degree of overlap between clusters on the attribute variables are reported beneath table 4 . These were generally large $(>0.8)$ representing good separation. ${ }^{42}$ There were no significant differences between the clusters in terms of age $[\mathrm{F}(2,82)=1.59]$, education $[\mathrm{F}(2,82)=0.39]$, predicted FSIQ $[\mathrm{F}(2,82)=2.26]$, and gender distribution $\left(\chi^{2}=0.20\right)$. In addition, there were no between cluster differences in the magnitude of pre- to postoperative change (SRB z scores) on measures of depression $[F(2,74)=2.01]$, anxiety $[F(2,74)=0.59]$, and stress $[F(2,74)=0.64]$ with an overall trend toward improved mood state

Analyses of the SRB z scores obtained by the three clusters on non-attribute variables are presented in table 4 . Significant between group main effects were found on two of six variables (TMT B/A, DSym). On TMT B/A post hoc analyses revealed cluster 3 (encoding/storage deficit) showed a significantly larger postoperative decrement compared to the other two clusters and the control group. This finding is contrary to the expectation that patients with a retrieval memory deficit (that is, cluster 2) would evince a concurrent decrement on measures of executive function. It is also noteworthy that no group differences emerged on the COWAT suggesting that performance on this executive task is dissociable from TMT B/A.

Both memory impaired clusters (2 and 3) showed a significant postoperative decrement on DSym compared to the control group. The memory spared subgroup (cluster 1) did not differ from any other group. This supports the view that DSym is a sensitive but non-specific indicator of acquired brain dysfunction. ${ }^{43}$

Finally, and again contrary to expectation, no group differences emerged on measures of confrontation naming (BNT) and manual dexterity (PegsRL) on which the encoding/storage deficit subgroup (cluster 3) and the retrieval deficit subgroup (cluster 2), respectively, were hypothesised to show the greatest postoperative decrement.

\section{Cardiac surgical variables}

Between group analyses of the preoperative medical and cardiac surgical data of the three clusters revealed no significant main effect on any variable (table 5). A main effect for the number of intraoperative perfusionist interventions approached significance $(p=0.058)$ with post hoc analysis revealing a marginally significant difference between the retrieval and encoding/storage deficit subgroups $(\mathrm{p}=0.045)$. However, given the total of 13 comparisons this result is likely due to chance.

Further correlational analyses were undertaken to identify any relationship between preoperative medical and cardiac surgical variables and pre- to postoperative change (SRB z scores) on the attribute variables (\%SDFR, Recognition) and the two non-attribute variables on which significant differences were found between clusters (TMT B/A, DSym). Only one of the 52 univariate correlations performed (4 neuropsychological measures $\times 13$ medical/surgical variables) was significant, this being an association between a positive history of hypertension and greater postoperative decrement

Table 3 Regression equations for predicting retest neuropsychological and mood scores

\begin{tabular}{|c|c|c|c|c|c|c|}
\hline \multirow[b]{2}{*}{ Measure } & \multirow[b]{2}{*}{$\mathbf{R}$} & \multirow[b]{2}{*}{$\mathrm{SE}_{\text {reg }}$} & \multirow[b]{2}{*}{ Intercept } & \multicolumn{3}{|c|}{ Significant unstandardised $\beta$ coefficients } \\
\hline & & & & $\boldsymbol{\beta}$ (baseline) & $\beta$ (age) & $\boldsymbol{\beta}$ (gender)* $^{*}$ \\
\hline \multicolumn{7}{|l|}{ CVLT } \\
\hline$\%$ SDFR & 0.60 & 13.78 & 35.47 & 0.66 & & \\
\hline Recognition & 0.57 & 1.08 & 9.11 & 0.41 & & \\
\hline \multicolumn{7}{|c|}{ Non-memory measures } \\
\hline BNT & 0.86 & 2.02 & 11.22 & 0.82 & & \\
\hline COWAT & 0.82 & 6.36 & 9.92 & 0.78 & & \\
\hline TMT B/A & 0.36 & 0.67 & 1.40 & 0.40 & & \\
\hline DSym & 0.87 & 4.78 & 24.4 & 0.89 & -0.22 & \\
\hline PegsRL & 0.82 & 1.14 & 1.45 & 0.75 & & 1.03 \\
\hline \multicolumn{7}{|l|}{ Mood } \\
\hline Depression & 0.80 & 2.32 & 0.72 & 0.76 & & \\
\hline Anxiety & 0.56 & 2.68 & 0.84 & 0.54 & & \\
\hline Stress & 0.69 & 4.11 & 2.23 & 0.60 & & \\
\hline
\end{tabular}


Table 4 Mean SRB z scores of cluster subtypes on neuropsychological measures

\begin{tabular}{|c|c|c|c|c|c|}
\hline $\begin{array}{l}\text { Neuropsychological } \\
\text { measure }\end{array}$ & $\begin{array}{l}\text { Cluster } 1 \text {, memory } \\
\text { spared }(n=41)\end{array}$ & $\begin{array}{l}\text { Cluster 2, retrieval } \\
\text { deficit }(n=30)\end{array}$ & $\begin{array}{l}\text { Cluster } 3 \text {, encoding } \\
\text { deficit }(n=14)\end{array}$ & Critical valuet & Post hoc \\
\hline \multicolumn{6}{|l|}{ Attribute variables } \\
\hline$\%$ SDFR & $0.27(0.79)$ & $-2.08(0.85)$ & $-0.84(1.06)$ & $66.74^{* * *}$ & $2 v C^{* * *}, 3 v C^{*}$ \\
\hline Recognition & $0.15(0.59)$ & $0.23(0.58)$ & $-1.81(0.70)$ & $32.56^{\star * *}$ & $3 v C^{* * *}$ \\
\hline \multicolumn{6}{|l|}{ Non-attribute variables } \\
\hline BNT & $-0.49(1.26)$ & $-0.26(0.97)$ & $-0.65(1.04)$ & 0.19 & \\
\hline TMT B/A & $-0.16(1.11)$ & $-0.12(1.26)$ & $-1.24(1.82)$ & $4.02^{* *}$ & $1 v 3^{*}, 2 v 3^{*}, 3 v C^{* * *}$ \\
\hline COWAT & $-0.34(1.00)$ & $-0.03(1.26)$ & $-0.45(1.62)$ & 3.14 & \\
\hline DSym & $-0.64(1.04)$ & $-1.00(1.33)$ & $-1.02(1.48)$ & $5.78^{* * *}$ & $2 v C^{* * *}, 3 v C^{*}$ \\
\hline PegsRL & $-0.69(1.21)$ & $-0.47(1.48)$ & $-0.14(1.61)$ & 7.17 & \\
\hline
\end{tabular}

C, control group. Control subjects' mean SRB z scores are not presented because they represent a normal distribution with a mean of 0 and an SD of 1.0. For attribute variables post hoc comparisons are only made between the control group and the cluster subgroups because, by definition, cluster analysis aims to create subgroups with minimal overlap. Nevertheless, for descriptive purposes between cluster effect sizes [Cohen's d, (\% overlap)] were calculated for the attribute variables. For clusters $1 \vee 2,1 \vee 3$, and $2 \vee 3$ these were \%SDFR: $2.9(<8.8 \%), 1.3(34.7 \%)$, and $1.4(31.9 \%)$, respectively, and for Recognition: 0.1 (92.3\%), 3.2 $(5.8 \%)$, and $3.3(<4.7 \%)$, respectively.

†One way analysis of variance (post hoc Tukey HSD): TMT B/A, DSym; otherwise Kruskal-Wallis tests (post hoc Mann-Whitney U tests); ${ }^{*} p<0.05$; ${ }^{* *} \mathrm{p}<0.01$; $* * * \mathrm{p}<0.001$.

in recognition memory ( $\mathrm{rho}=0.24, \mathrm{p}=0.03$ ). Again, this single significant result is likely due to chance given the large number of comparisons performed.

Finally, preoperative medical and cardiac surgical variables were examined against the number of cognitive domains (episodic memory, naming, executive, psychomotor) on which patients demonstrated statistically significant declines (that is, SRB z scores below -1.64). Twenty six patients (30.6\%) were not impaired on any domain and 37 (43.5\%), 16 $(18.8 \%)$, and $6(7.1 \%)$ were impaired on 1,2 , and $\geqslant 3$ domains, respectively. Once again, no between group differences were found on any of the medical or surgical variables.

\section{DISCUSSION}

This study identified three subtypes of memory outcome following CABG: memory spared (48\% of patients), retrieval deficit (35\%), and encoding/storage deficit (17\%). However, contrary to the cortical/subcortical neurobehavioral models, it was found that the retrieval and encoding deficit clusters generally did not display concurrent patterns of spared and impaired cognitive function typically associated with these syndromes. In fact, on several measures, the two clusters were indistinguishable from each other as well as being indistinguishable from the memory spared cluster and control participants. In the broadest of terms these findings challenge the notion of cortical and subcortical dementia being neuropsychologically dissociable syndromes. Alternately, at the very least, the utility of these syndromes in defining subtypes of neuropsychological outcome following CABG surgery is brought into question. As will be discussed, this may be because of insufficient overlap between the structures injured in CABG surgery and those involved in the production of prototypic cortical and subcortical neurobehavioral syndromes. In addition, the limitations of cluster analysis in defining distinct patient subgroups must be considered.

The failure of the retrieval deficit subgroup to display the full spectrum of neuropsychological deficits typically associated with a subcortical syndrome suggests that the individual features comprising the syndrome do not share a common neuroanatomic substrate. Given that five parallel but segregated basal ganglia-thalamocortical circuits have been identified, which are hypothesised to subserve different aspects of behaviour and cognition, ${ }^{22}$ this finding is not surprising. However, within the context of the present study the issue remains as to which systems are selectively injured and spared in CABG surgery resulting in the production of a memory retrieval deficit without concurrent alteration in executive function and manual dexterity. It is noteworthy

Table 5 Preoperative medical and cardiac surgical characteristics of CABG surgery cluster subtypes

\begin{tabular}{|c|c|c|c|c|}
\hline \multirow[b]{2}{*}{ Variable } & \multicolumn{3}{|l|}{ Cluster subgroup } & \multirow[b]{2}{*}{ Critical value } \\
\hline & Memory spared $(n=41)$ & Retrieval deficit $(n=30)$ & Encoding deficit $(n=14)$ & \\
\hline \multicolumn{5}{|l|}{ Preoperative } \\
\hline Hypertension (yes/no) & $16 / 21$ & $14 / 15$ & $9 / 4$ & $2.62 \dagger$ \\
\hline Diabetes (yes/no) & $7 / 30$ & $5 / 24$ & $2 / 11$ & $0.90 t$ \\
\hline LVF (normal/altered/poor) & $21 / 9 / 5$ & $18 / 7 / 1$ & $9 / 5 / 0$ & $3.98 t$ \\
\hline CCS score $(\leqslant 2 / \geqslant 3)$ & $13 / 19$ & $15 / 13$ & $8 / 5$ & $1.95 t$ \\
\hline \multicolumn{5}{|l|}{ Surgical } \\
\hline Grafts & $2.4(0.7)$ & $2.3(0.8)$ & $2.6(1.0)$ & 0.61 \\
\hline Cross clamp time (min) & $23.2(9.0)$ & $22.4(7.9)$ & $26.1(9.7)$ & 0.84 \\
\hline CPB time (min) & $42.8(15.5)$ & $41.9(15.2)$ & 45.7 (17.5) & 0.28 \\
\hline $\mathrm{MCCP}(\mathrm{mm} \mathrm{Hg})$ & $42.4(6.2)$ & $42.5(4.5)$ & $39.9(5.4)$ & 1.30 \\
\hline Mflow (l//min) & $3.8(0.4)$ & $3.8(0.4)$ & $3.9(0.5)$ & 0.36 \\
\hline Interventions & $5.7(2.8)$ & $4.9(2.2)$ & $7.1(4.1)$ & 2.96 \\
\hline Hypothermic/normothermic & $19 / 22$ & $17 / 13^{2 /}$ & $6 / 8$ & $0.90 \dagger$ \\
\hline Rhythm (yes/no) & $29 / 12$ & $24 / 6$ & $11 / 3$ & $1.03 \dagger$ \\
\hline ICU stay $(\mathrm{h})$ & $31.4(16.1)$ & $32.7(23.0)$ & 34.4 (22.9) & 0.13 \\
\hline
\end{tabular}

CCS score, Canadian Cardiovascular Society score ( $\mathrm{n}=73$ ); CPB time, cardiopulmonary bypass time; diabetes, $\mathrm{n}=79$; hypertension, $\mathrm{n}=79$; Interventions, number of perfusionist interventions; LVF, left ventricular function $(n=75)$; MCPP, mean cerebral perfusion pressure; Mflow, mean blood flow rate; Rhythm, spontaneous return to sinus rhythm.

$\mathrm{t} \chi^{2}$ test (asymptotic two sided), $d f=2$ (except $\left.L V F d f=4\right)$. All other comparisons one way ANOVA $F(2,82)$, except $M A P F(2,73)$ and ICU stay $F(2,80)$. ${ }^{*}$ All $p>0.05$. 
here that these aspects of neuropsychological functioning previously have been found to be dissociated in nondemented individuals with deep white matter hyperintensities. $^{44} 45$ This suggests that "incomplete" forms of the subcortical syndrome may be found when pathology is less severe and extensive.

The finding that CABG patients within the encoding/ storage deficit cluster did not display a significant concurrent decrement in confrontation naming as in prototypic cortical dementia suggests that the neocortical structures usually associated with this loss remained spared. In fact, the finding that this subgroup actually showed an unexpected postsurgical decrement on a measure of executive ability (TMT B/A) raises the question of frontal/subcortical involvement. Specifically, given that lesions within diencephalic structures such as the dorsomedial nucleus of the thalamus and mamillothalamic tract produce memory disturbance qualitatively similar to that seen with hippocampal damage in addition to producing subcortical features ${ }^{46}$ it is conceivable that damage to these structures could account for the findings in this subgroup. Moreover, because thalamic nuclei are supplied by small penetrating arteries, they may be especially vulnerable to intraoperative microembolic injury.

The finding that the memory spared cluster did not show significantly greater postoperative decrements compared to either of the other two clusters or control subjects on measures of executive or psychomotor function suggests that the memory variables used in establishing cluster subtypes were also good general markers of neuropsychological integrity. However, what cannot be determined from this study, which focused on subtypes of episodic memory dysfunction, is whether the memory spared cluster contained a subset of patients with a constellation of deficits that did not include memory dysfunction. For example, inspection of Table 4 reveals that compared to the other clusters the memory spared cluster showed the largest, albeit nonsignificant, mean postoperative decline on PegsRL (SRB $\mathrm{z}=-0.69$ ). Thus, it is conceivable that this cluster contained some patients with acquired selective deficits in psychomotor function possibly due to damage restricted to the subcortical motor circuit..$^{22}$ Identification of a memory spared but otherwise impaired subgroup of patients awaits further study.

Finally, the failure to observe the classically described syndromes of cortical and subcortical dementia following CABG surgery might relate to the limitations of cluster analysis in identifying individuals with "pure" forms of these syndromes. Specifically, as noted by Beatty et al, ${ }^{47}$ "....cluster analysis will always produce clusters and the average performance of participants in a cluster may not be representative of all or even most of the subjects in the cluster" (p. 60). This being the case, future research could maximise separation of patients with pure cortical and subcortical (or mixed) syndromes by selecting only those patients showing statistically significant postoperative decrements (that is, SRB z score $<-1.64$ ) on the relevant combinations of neuropsychological measures. However, in order to identify sufficient numbers of these "pure" cases a much larger patient sample than that used in the present study is required in which only $7 \%$ of patients showed statistically significant decrements across three or more domains. Nevertheless, such an approach would provide a more powerful means of determining whether qualitatively distinct postoperative neuropsychological syndromes are the products of different pathophysiological mechanisms.

\section{Cardiac surgical variables}

Notwithstanding the limitations of cluster analysis, there are three potential explanations to account for the failure to observe hypothesised between cluster differences on intraoperative physiologic measures. First, the measures used might have been insensitive markers of intraoperative neurologic insult and more direct measures such as transcranial Doppler measurement of microembolic delivery might have disclosed between cluster differences. Second, irrespective of the sensitivity of the measures employed, the simple univariate between cluster comparisons that were undertaken might have failed to disclose potential complex interactions between the various measures. For example, poor neuropsychological outcome due to microembolism might have been predicted by a combination of a higher number of perfusionist interventions, longer duration of CPB, and low cerebral flow rate impeding embolic washout. ${ }^{48}$ This may have been further moderated by increasing age. ${ }^{11}$

Finally, it is conceivable that the three clusters actually were subjected to the same type and degree of intraoperative insult with the extent and nature of their postoperative neuropsychological sequelae being a product of this equal insult upon brains with varying degrees and locations of preexisting damage. This hypothesis engages the emerging theoretical concept of "brain reserve capacity" ${ }^{\prime 49}$ which serves to explain different neuropsychological outcomes amongst individuals sustaining an equivalent level of injury. Support for this concept of patients being "primed" for post-CABG neuropsychological dysfunction comes from a report showing that patients demonstrating postoperative neuropsychological decline had significantly poorer pre- and intraoperative cerebral metabolism on SPECT scanning than did patients without SPECT abnormalities. ${ }^{50}$ Presumably, the anatomic location of any such preoperative damage determines the quality and pattern of neuropsychological deficits that emerge postoperatively. In other words, brain integrity prior to surgery may be more important in determining postoperative neuropsychological outcome than the type and degree of intraoperative hazards to which the brain is exposed. This hypothesis awaits testing by comparing the neuropsychological outcomes of patients with varying degrees and locations of preoperative MRI defined pathology but who are matched on intraoperative physiologic measures considered to be markers of cerebral insult.

In conclusion, this study identified three subtypes of neuropsychological outcome following CABG surgery using a theoretically driven approach to define pre- to postoperative change. Although the patterns of neuropsychological change did not tightly conform to established prototypic syndromes, the emergence of neuropsychologically heterogeneous subgroups nevertheless challenges the appropriateness of the common practice of collapsing individual test scores to arrive at a single incidence figure of "impairment". ${ }^{1}$ In this respect the present study serves as a starting point for future work in establishing whether CABG surgery imparts a unique pattern (or patterns) of neuropsychological compromise that may relate to the vulnerability of specific neural structures to intraoperative injury. From a clinical perspective, an appreciation of the fact that CABG surgery does not impart a unitary neuropsychological deficit provides for greater understanding of patients' complaints and likely real world consequences.

\section{ACKNOWLEDGEMENTS}

The authors thank Marie Andrew, BA(Hons) for testing CABG patients.

\section{Authors' affiliations \\ A C Kneebone, M A Luszcz, School of Psychology, Flinders University of South Australia, Adelaide, Australia \\ R A Baker J L Knight, Cardiac Surgery Research Unit, Flinders Medical Centre, Adelaide, Australia}


This study was supported by the National Heart Foundation of Australia (Grant\# G95A4458)

Competing interests: none declared

\section{REFERENCES}

1 Mahanna EP, Blumenthal JA, White WD, et al. Defining neuropsychological dysfunction after coronary artery bypass grafting. Ann Thorac Surg 1996;61:1342-7.

2 Witoszka MM, Tamura H, Indeglia R, et al. Electroencephalographic changes and cerebral complications in open-heart surgery. J Thorac Cardiovasc Surg 1973:66:855-64.

3 Tufo HM, Ostfeld AM, Shekelle R. Central nervous system dysfunction following open-heart surgery. JAMA 1970;212:1333-40.

4 Malone M, Prior P, Scholtz CL. Brain damage after cardiopulmonary bypass: correlations between neurophysiological and neuropathological findings. J Neurol Neurosurg Psychiatry 1981;44:924-31.

5 Sylivris S, Levi C, Matalanis G, et al. Pattern and significance of cerebral microemboli during coronary artery bypass grafting. Ann Thorac Surg 1998;66:1674-8.

6 Pullicino PM, Bhayana G, Lajos $T$, et al. White matter hyperintensities and hypotension in coronary bypass surgery. Cerebrovasc Dis 1992;2:230.

7 Steinberg GK, De La Paz R, Mitchell RS, et al. MR and cerebrospinal fluid enzymes as sensitive indicators of subclinical cerebral injury after open-heart valve replacement surgery. AJNR Am J Neuroradiol 1996;17:205-12.

8 Toner I, Peden CJ, Hamid SK, et al. Magnetic resonance imaging and neuropsychological changes after coronary artery bypass graft surgery: preliminary findings. J Neurosurg Anesthesiol 1994;3:163-9.

9 Vanninen R, Aikia M, Kononen M, et al. Subclinical cerebral complications after coronary artery bypass grafting: prospective analysis with magnetic resonance imaging, quantitative electroencephalography, and neuropsychological assessment. Arch Neurol 1998;55:618-27.

10 Restrepo L, Wityk RJ, Grega MA, et al. Diffusion- and perfusion-weighted magnetic resonance imaging of the brain before and after coronary artery bypass grafting surgery. Stroke 2002;33:2909-15.

11 Bendszus $M$, Reents W, Franke D, et al. Brain damage after coronary artery bypass grafting. Arch Neurol 2002;59:1090-5.

12 Cummings JL. Subcortical dementia: neuropsychology, neuropsychiatry, and pathophysiology. Br J Psychiatry 1986;149:682-97.

13 Delis DC, Massman PJ, Butters N, et al. Profiles of demented and amnesiac patients on the California Verbal Learning Test: implications for the assessment of memory disorders. Psychol Assess 1991;3:19-26.

14 Braak H, Braak E. Neuropathological staging of Alzheimer-related changes. Acta Neuropathol 1991;82:239-59.

15 McPherson SE, Cummings JL. Neuropsychological aspects of vascular dementia. Brain Cogn 1996;31:269-82.

16 Libon DJ, Bogdanoff B, Bonavita J, et al. Dementia associated with periventricular deep white matter alterations: a subtype of subcortical dementia. Arch Clin Neuropsychol 1997;12:239-50.

17 Mendez MF, Ashla-Mendez M. Differences between multi-infarct dementia and Alzheimer's disease on unstructured neuropsychological tasks. J Clin Exp Neuropsychol 1991;13:923-32.

18 Pillon B, Deweer B, Agid Y, et al. Explicit memory in Alzheimer's, Huntington's, and Parkinson's diseases. Arch Neurol 1993:50:374-9.

19 Libon DJ, Mattson RE, Glosser G, et al. A nine-word dementia version of the California Verbal Learning Test. Clin Neuropsychol 1996;10:237-44.

20 Hodges JR, Patterson K. Is semantic memory consistently impaired early in the course of Alzheimer's disease? Neuroanatomical and diagnostic implications. Neuropsychologia 1995;33:441-59.

21 Looi JCL, Sachdev PS. Differentiation of vascular dementia from AD on neuropsychological tests. Neurology 1999;53:670-8.

22 Darvesh S, Freedman M. Subcortical dementia: a neurobehavioral approach Brain Cogn 1996;31:230-49.

23 Almkvist O, Bäckman L, Basun H, Wahlund L-O. Patterns of neuropsychological performance in Alzheimer's disease and vascular dementia. Cortex 1993;29:661-73
24 Kertesz A, Clydesdale S. Neuropsychological deficits in vascular dementia vs Alzheimer's disease: frontal lobes deficits prominent in vascular dementia. Arch Neurol 1994;51:1226-31.

25 Taylor RL, Borger MA, Weisel RD, et al. Cerebral microemboli during cardiopulmonary bypass: increased emboli during perfusionist interventions. Ann Thorac Surg 1999;68:89-93.

26 Brown WR, Moody DM, Challa VR, et al. Longer duration of cardiopulmonary bypass is associated with greater numbers of cerebral microemboli. Stroke 2000;31:707-13.

27 Murkin JM, Newman SP, Stump DA, et al. Statement of consensus on assessment of neurobehavioral outcomes after cardiac surgery. Ann Thorac Surg 1995;59:1289-95.

28 Klafta JM, Zacny JP, Young CJ. Neurological and psychiatric adverse effects of anaesthetics. Drug Saf 1995;13:281-95.

29 Heyer EJ, Sharma R, Winfree CJ, et al. Severe pain confounds neuropsychological test performance. J Clin Exp Neuropsychol 2000;22:633-9.

30 Delis DC, Kramer JH, Kaplan E, et al. California Verbal Learning Test Manual. San Antonio, TX: The Psychological Corporation, 1987.

31 Tiffin J. Purdue Pegboard examiner's manual. Rosemont, IL: London House, 1968.

32 Borkowski JG, Benton AL, Spreen O. Word fluency and brain damage. Neuropsychologia 1967:5:135-40.

33 Armitage SG. An analysis of certain psychological tests used for the evaluation of brain injury. Psychol Monogr, 1946;60 (Whole No.277).

34 Kaplan EP, Goodglass H, Weintraub S. The Boston Naming Test, 2nd ed. Philadelphia, PA: Lea \& Febiger, 1983.

35 Wechsler D. Wechsler Adult Intelligence Scale-Revised: Manual. New York: Psychological Corporation, 1981

36 Crawford JR. Current and premorbid intelligence measures in neuropsychological assessment. In: Crawford JR, Parker DM, McKinlay W, eds. A handbook of neuropsychological assessment. London: Erlbaum, 1992:267-91.

37 Lovibond SH, Lovibond PF. Manual for the Depression Anxiety Stress Scales. Sydney: The Psychology Foundation of Australia, 1995.

38 Arbuthnott K, Frank J. Trail Making Test, Part B as a measure of executive control: validation using a set switching paradigm. J Clin Exp Neuropsychol 2000;22:518-28

39 Sawrie SM, Chelune GJ, Naugle RI, et al. Empirical methods for assessing meaningful change following epilepsy surgery. J Int Neuropsychol Soc 1996:2:556-64.

40 Everitt B. Cluster analysis, 2nd ed. Aldershot, UK: Gower, 1980

41 Mojena R. Hierarchical grouping methods and stopping rules: an evaluation. Comput J 1977;20:359-63.

42 Cohen J. Statistical power analysis for the behavioral sciences, 2nd ed. New York: Academic Press, 1988:25-6.

43 Lezak MD. Neuropsychological assessment, 3rd ed. New York: Oxford University Press, 1995:120.

44 Gunning-Dixon FM, Raz N. The cognitive correlates of white matter abnormalities in normal aging: a quantitative review. Neuropsychology 2000; 14:224-32.

45 Boone K, Miller BL, Lesser IM, et al. Neuropsychological correlates of whitematter lesions in healthy elderly subjects. Arch Neurology 1992;49:549-54.

46 Schmahmann JD. Vascular syndromes of the thalamus. Stroke 2003:34:2264-78.

47 Beatty WW, Wilbanks SL, Blanco CR, et al. Memory disturbances in multiple sclerosis: reconsideration of patterns of performance on the Selective Reminding Test. J Clin Exp Neuropsychol 1996;18:56-62.

48 Caplan LR, Hennerici M. Impaired clearance of emboli (washout) is an important link between hypoperfusion, embolism, and ischemic stroke. Arch Neurol 1998;55:1475-82

49 Satz P. Brain reserve capacity on symptom onset after brain injury: a formulation and review of evidence for threshold theory. Neuropsychology 1993;7:273-95.

50 Hall RA, Fordyce DJ, Lee ME, et al. Brain SPECT imaging and neuropsychological testing in coronary artery bypass patients. Ann Thorac Surg 1999;68:2082-8. 